\title{
Actividad micoparasítica de nueve aislamientos de diferentes cepas de Trichoderma sp. sobre Rhizoctonia solani en frejol Caupí (Vigna unguiculata L.)
}

\section{Mycoparasitic activity of nine isolates of different strains of Trichoderma sp. on Rhizoctonia solani in Cowpea beans (Vigna unguiculata L.)}

\author{
Cynthia Garrido Flores ${ }^{1}$, Carolina Cedano Saavedra ${ }^{1}$, Martín Delgado Junchaya ${ }^{2}$ y Zinthia Neira \\ Sánchez ${ }^{1}$
}

'Universidad Nacional de Trujillo, Perú.

${ }^{2}$ Universidad Privada Antenor Orrego, Perú.

\begin{abstract}
RESUMEN
Rhizoctonia solani, fitopatógeno causante de "chupadera fungosa", afecta todas las plantas que se propagan por semilla botánica en las fases de germinación y plántula. El objetivo fue evaluar la actividad micoparasítica de nueve aislamientos de diferentes cepas de Trichoderma sobre la Rhizoctonia solani, utilizando frejol Caupí (Vigna unguiculata L). Los aislamientos fueron T20, T17, T9, T10, TSV2, TSV, T10' de Trichoderma asperellum; T5 de Trichoderma viride y T327 de Trichoderma harzianum. Se consideró un tratamiento solo con Rhizoctonia solani, como testigo de virulencia y un tratamiento solo con frejol Caupí como testigo de la viabilidad de la semilla. Los tratamientos fueron dispuestos en un diseño completo al azar con 10 repeticiones. Se empleó la técnica de enfrentamiento dual en medio de cultivo Papa-Dextrosa-Agar (PDA) en placa de Petri, considerándose la unidad experimental. Se tomaron muestras de la zona de colonización y se observaron al microscopio para comprobar el parasitismo. Se evaluó porcentaje de germinación, emergencia de plántulas e incidencia de chupadera fungosa. Trichoderma harzianum (T327) y Trichoderma asperelum (T17) presentaron mayor germinación y emergencia de plántulas de frejol Caupí y los porcentajes más bajos de incidencia de chupadera fungosa (3.25 y 5.29 respectivamente), teniendo actividad micoparasítica sobre Rhizoctonia solani, constituyéndose una alternativa biológica importante para su control.
\end{abstract}

Palabras clave: micoparasitismo, Rhizoctonia solani, Trichoderma sp, frejol Caupí, control. 
Rhizoctonia solani, a phytopathogen that causes "damping off", affects all plants that are propagated by botanical seed in the germination and seedling phases. The objective was to evaluate the mycoparasitic activity of nine isolates of different Trichoderma strains on Rhizoctonia solani, using Cowpea beans (Vigna unguiculata L). The isolates were 'T20, T17, T9, T10, TSV2, TSV, T10' from Trichoderma asperellum; T5 from Trichoderma viride and T327 from Trichoderma harzianum. A treatment only with Rhizoctonia solani was considered, as a virulence control and a treatment only with Cowpea beans as a control of the viability of the seed. The treatments were arranged in a complete randomized design with 10 repetitions. The dual confrontation technique was used in a potato-dextrose-agar (PDA) culture medium in a Petri dish, considering the experimental unit. Samples were taken from the colonization zone and observed under a microscope to verify parasitism. Percentage of germination, seedling emergence and incidence of fungus lick were evaluated. Trichoderma harzianum (T327) and Trichoderma asperelum (T17) presented higher germination and emergence of cowpea bean seedlings and the lowest percentages of incidence of fungus sucking ( 3.25 and 5.29 respectively), having mycoparasitic activity on Rhizoctonia solani, becoming an important biological alternative for its control.

Keywords: mycoparasitism, Rhizoctonia solani, Trichoderma sp, Cowpea beans, control.

\section{INTRODUCCIÓN}

$\mathrm{E}$ 1 frejol Caupí (Vigna unguiculata $L$ ) es una leguminosa importante en la alimentación humana, pues se consume como grano seco o en fresco y se siembra en muchas regiones tropicales y subtropicales del mundo. Esta especie es muy susceptible, en las etapas fenológicas de germinación y plántula, al ataque de Rhizoctonia solani Kühn, patógeno habitante común en los suelos agrícolas y causante de la enfermedad denominada "chupadera fungosa". En el Perú, esta enfermedad afecta a un gran número de cultivos, tales como: fréjol, cebada, algodón, tomate, papa, etc., causando daños serios durante cualquier estado de desarrollo de la planta (Arcos \& Zúñiga., 2015).

Los síntomas producidos en V. unguiculata por R. solani se observan desde la preemergencia, a manera de un estrangulamiento con lesiones necróticas en el cuello o pie de la planta, hasta en plantas de más edad, en las cuales las hojas inferiores se vuelven amarillas y el tallo muestra cancros de color rojo ladrillo, que pueden llegar a cubrir la raíz ocasionando la muerte de la planta. El control de este patógeno se realiza fundamentalmente con productos químicos o desinfectantes de semillas los cuales son formulados con diferentes ingredientes activos que vienen solos o en mezcla y son, en su mayoría, muy tóxicos por lo que presentan desventajas ambientales y económicas (Infante et al., 2013; Reyes et al., 2016).

Debido a su residualidad, estos productos se acumulan en cuerpos de agua, suelo, plantas y animales; además, generan resistencia por parte de los fitopatógenos aunado al detrimento que causan en la salud humana (Rubio et al., 2008). Actualmente, existe gran preocupación por el desarrollo de alternativas que garanticen una mayor sostenibilidad en la producción agrícola, minimizando el impacto sobre el medio ambiente (Gonza Carnero et al., 2013; Guédez et al., 2009). En este contexto adquieren particular importancia los microorganismos con capacidad antagónica, entre los cuales es ampliamente conocido el género Trichoderma, cuyas especies pueden ser usadas como promisorios agentes de control biológico (Michel, 2001; Hoyos-Carvajal et al., 2008; Esparza, 2009; Barahona, 2012). Attribution-NonCommercial 4.0 International (CC BY-NC 4.0)
Las diferentes especies de Trichoderma ejercen actividad antagónica por competencia de nutrientes o espacio, por antibiosis (producción de metabolitos), modificando las condiciones ambientales o mediante la producción de sustancias promotoras del crecimiento vegetal y de forma directa por micoparasitismo (Tovar, 2008; Capouz, 2009; Infante et al., 2010; Esquivel, 2012; Martínez et al., 2013). El objetivo de esta investigación fue evaluar la actividad micoparasítica de nueve aislamientos de diferentes cepas de Trichoderma sp. en el control de Rhizoctonia solani en frejol Caupí (Vigna unguiculata L.).

\section{Materiales Y MétodoS}

\section{Cepas de Trichoderma sp.}

Para realizar la presente investigación se contó con los aislamientos T20, T17, T09, T10, TSV2, TSV y T10' de Trichoderma asperellum; $\mathrm{T} 5$ de Trichoderma viride; procedentes de la micoteca de la Empresa Agroindustrial Camposol S.A. y un aislamiento codificado como T327 de Trichoderma harzianum.

\section{Condiciones de crecimiento de Trichoderma sp. y Rhizoctonia solani}

Los aislamientos de Trichoderma sp. y Rhizoctonia solani empleados en este estudio fueron desarrollados en placas de Petri con medio de cultivo a base de Papa-Dextrosa-Agar (PDA) al $2 \%$ e incubados a temperatura ambiente $\left(24^{\circ} \mathrm{C}\right)$ durante siete días.

\section{Evaluación del potencial antagónico de Trichoderma sp. sobre Rhizoctonia solani}

Para evaluar la actividad micoparásitica de Trichoderma $s p$. sobre Rhizoctonia solani, se utilizó el método de cultivo dual en placas de Petri con PDA al $2 \%$, colocando discos de $8 \mathrm{~mm}$ de diámetro colonizado con micelio de Trichoderma en un extremo de la placa y discos con micelio de $R$. solani en el extremo opuesto. Las placas fueron incubadas a temperatura ambiente y se observaron diariamente hasta determinar el nivel de colonización ejercido por Trichoderma sobre el micelio de $R$. solani $o$ el desarrollo de ambos.

Se utilizó un diseño completo al azar (DCA) con 11

https://doi.org/10.46908/tayacaja.v4i2.172 dol pág. 60 
tratamientos: nueve tratamientos consistieron en aislamientos distintos de Trichoderma y dos tratamientos testigo de los cuales, uno fue solo $R$. solani, para validar su patogenicidad y el otro fue frejol Caupi sembrado en suelo estéril, para demostrar la viabilidad de la semilla. Cada tratamiento tuvo 10 repeticiones, que consistieron en una placa con 10 semillas, constituyendo la unidad experimental.

El impacto del antagonista sobre el micelio de $R$. solani, se evaluó in vivo, utilizando semillas de frejol Caupí, como hospedero susceptible a este fitopatógeno. Para ello, las placas del cultivo dual se llenaron con tierra estéril y luego se sembraron 10 semillas de frejol Caupí, en cada una. Las placas se regaron, según requerimiento, con agua destilada estéril y fueron mantenidas a temperatura ambiente durante 25 días (Fig. 1).

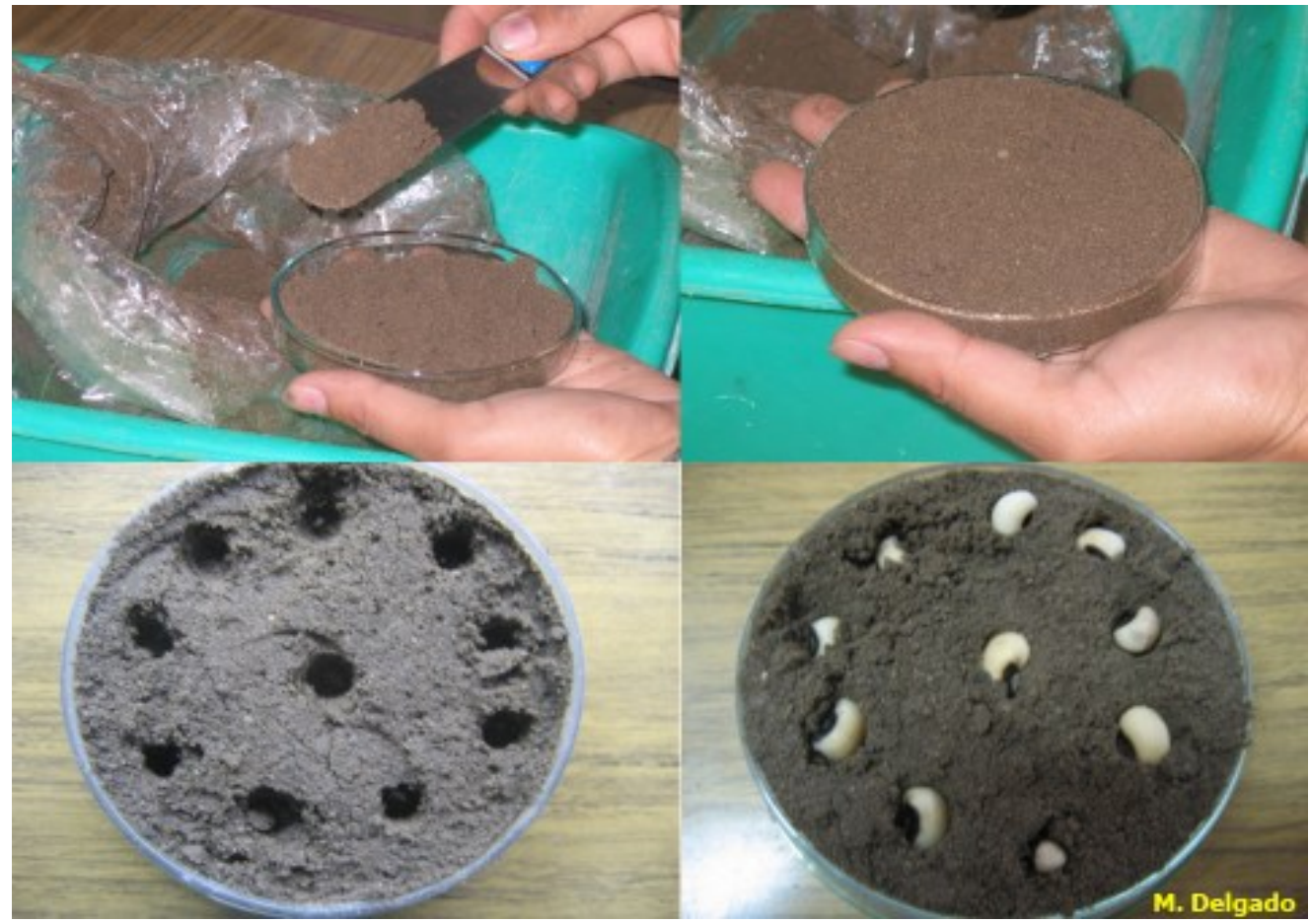

\section{Figura 1.}

Siembra de semillas de frejol Caupí para evaluar la viabilidad del micelio de Rhizoctonia solani después de la colonización por diferentes aislamientos de Trichoderma $s p$.

\section{Parámetros evaluados}

Porcentaje de germinación y emergencia de plántulas

\section{Ganancia de peso}

En cada repetición se contó el número de semillas germinadas y número de plántulas emergidas obteniéndose el promedio que se expresó en porcentaje.

\section{Incidencia de chupadera fungosa}

Se determinó contando el número de plantas afectadas, para lo cual se extrajeron las plántulas que germinaron en cada placa y se procedió a evaluar la presencia de lesiones necróticas, hundidas o deprimidas de color marrón oscuro (estrangulamiento) en la zona del cuello o base del tallo de la plántula. Los resultados obtenidos se expresaron en porcentaje.

\section{Micoparasitismo}

Antes de la siembra del frejol Caupí se extrajeron pequeñas porciones de medio PDA de la zona de interacción entre Trichoderma sp. y R. solani y se prepararon montajes sobre láminas cubre y porta objeto adicionados con lactofenol y se observaron al microscopio compuesto de luz para determinar si el micelio de $R$. solani presentaba lisis o fragmentación debido al parasitismo de Trichoderma.

Las evaluaciones se realizaron durante 12 días y los datos obtenidos fueron comparados mediante el análisis de varianza (ANOVA) y la prueba de Tukey con un nivel de significancia estadístico $\mathrm{p}:<0.05$.

\section{Resultados}

\section{Porcentaje de germinación y emergencia de plántulas}

Los análisis de varianza (ANOVA) del porcentaje de germinación y emergencia de plántulas indican que el efecto de los aislamientos de Trichoderma sobre Rhizoctonia solani fue altamente significativo ( $\mathrm{p}:<0.05)$.

En la Tabla 1, se muestran los resultados de la prueba de medias de Tukey de los datos correspondientes al porcentaje de germinación obtenido en cada tratamiento del experimento. Se observa que todos los aislamientos de Trichoderma tuvieron un efecto similar sobre el control de Rhizoctonia solani, favoreciendo la germinación de frejol Caupí en un rango promedio de 90.5 a $94.3 \%$, a diferencia del tratamiento solo con Rhizoctonia solani donde no hubo germinación debido al severo ataque del fitopatógeno $(0 \%)$. 
Tabla 1

ISSNe: $2617-9156$

Porcentaje de germinación de semillas de frejol Caupí sembradas en las placas del cultivo dual de Trichoderma sp. vs Rhizoctonia solani.

\begin{tabular}{|c|c|c|c|}
\hline Tratamiento & $\%$ & Gern & inación \\
\hline Frejol Caupí & 94.4 & $\mathrm{a}$ & \\
\hline Trichoderma viride T327 & 94.3 & $\mathrm{a} \quad \mathrm{b}$ & \\
\hline Trichoderma asperellum T17 & 93.9 & $\mathrm{a} \quad \mathrm{b}$ & $\mathrm{c}$ \\
\hline T. asperellum TSV10' & 93.6 & $a \quad b$ & $\mathrm{c}$ \\
\hline $\mathrm{T}$. asperellum $\mathrm{T} 10$ & 93.0 & $a \quad b$ & $\mathrm{c}$ \\
\hline $\mathrm{T}$. viride $\mathrm{T} 5$ & 93.2 & $a \quad b$ & $\mathrm{c}$ \\
\hline T. asperellum T09 & 92.5 & $\mathrm{a} \quad \mathrm{b}$ & $\mathrm{c}$ \\
\hline T. asperellum TSV & 91.2 & $\mathrm{a} \quad \mathrm{b}$ & $\mathrm{c}$ \\
\hline T. asperellum T20 & 90.8 & b & $\mathrm{c}$ \\
\hline T. asperellum TSV2 & 90.5 & & $\mathrm{c}$ \\
\hline Rhizoctonia solani & 0 & & $\mathrm{~d}$ \\
\hline
\end{tabular}

Las letras similares significan que los valores no son estadísticamente diferentes

Tukey: p: $<0.05$

La Tabla 2, muestra los resultados de la prueba de medias de Tukey correspondientes al porcentaje de emergencia de plántulas. Se observa que todos los aislamientos de Trichoderma tuvieron un efecto similar sobre el control de $R$. solani, favoreciendo la emergencia de las plántulas en un rango promedio de 85.5 a $89 \%$, a diferencia del tratamiento de Rhizoctonia solani donde fue totalmente inhibida la emergencia (0\%).

\section{Tabla 2}

Porcentaje de emergencia de plántulas de frejol Caupí.

\begin{tabular}{lc}
\hline \multicolumn{1}{c}{ Tratamiento } & \% Emergencia \\
\hline Frejol Caupí & 89.0 \\
Trichoderma viride T327 & 89.0 \\
Trichoderma asperellum T17 & 88.9 \\
T. asperellum TSV10' & 88.2 \\
T. asperellum T10 & 88.0 \\
T. viride T5 & 87.2 \\
T. asperellum T09 & 86.7 \\
T. asperellum TSV & 85.9 \\
T. asperellum T20 & 85.8 \\
T. asperellum TSV2 & 85.5 \\
Rhizoctonia solani & $00.0 \quad$ a \\
\hline
\end{tabular}

Las letras en común significan que los valores no son estadísticamente diferentes.

\section{Incidencia de chupadera fungosa}

El análisis de varianza (ANOVA) del porcentaje de plántulas con chupadera fungosa (Incidencia) indica alta significancia $(\mathrm{p}<0,05)$ para tratamientos (aislamientos de

Trichoderma) con un C.V. de $18.3 \%$ y un R2 de 0.83 . La prueba de Tukey, expuesta en la Tabla 3, muestra que los tratamientos que tuvieron el menor porcentaje de plantas con chupadera fungosa fueron aquellos en los que Rhizoctonia solani fue enfrentado a Trichoderma harzianum aislamiento T327 (3.29\%) y a Trichoderma asperellum aislamiento T17 (5.25\%). En los demás aislamientos de Trichoderma se encontró diferentes porcentajes de incidencia de chupadera fungosa (15.50 a $51.25 \%)$. 
Tabla 3

ISSNe: $2617-9156$

Incidencia de chupadera fungosa en frejol Caupí, causada por Rhizoctonia solani, posterior a la exposición del parasitismo de nueve aislamientos de diferentes cepas de Trichoderma sp.

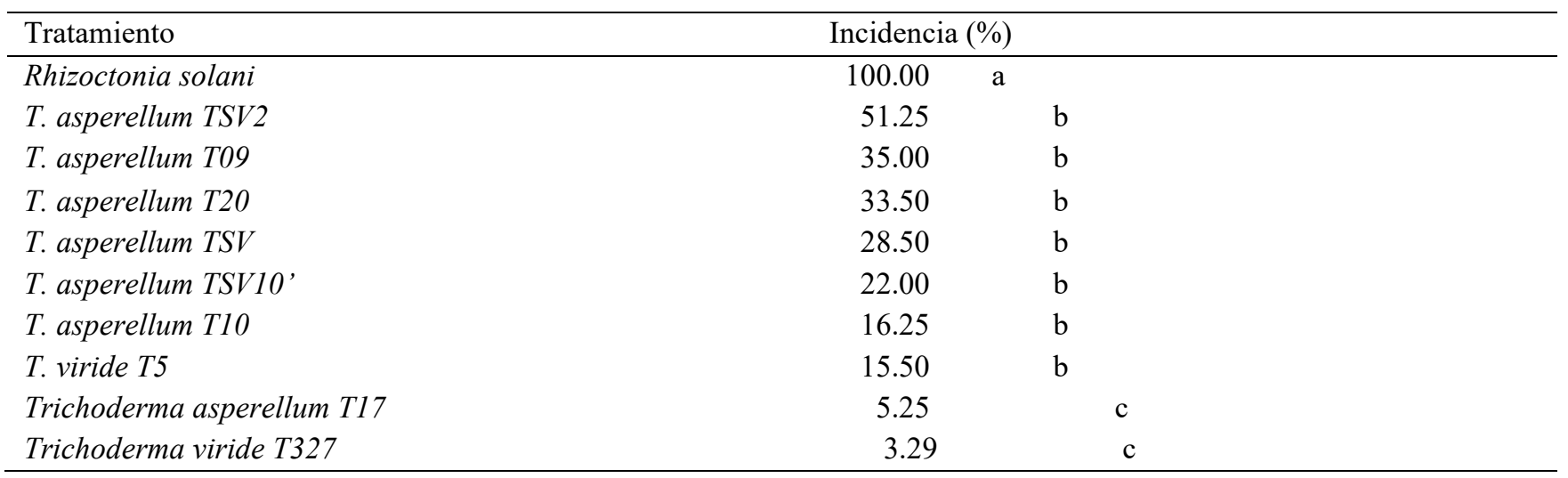

\section{Micoparasitismo}

Todos los aislamientos de Trichoderma sp. formaron gran cantidad de micelio y esporas, invadiendo totalmente la superficie de la placa Petri y cubriendo el área donde desarrolló Rhizoctonia solani, con lo cual mostraron el máximo efecto antagónico, según la escala de Bell et al. (1982).

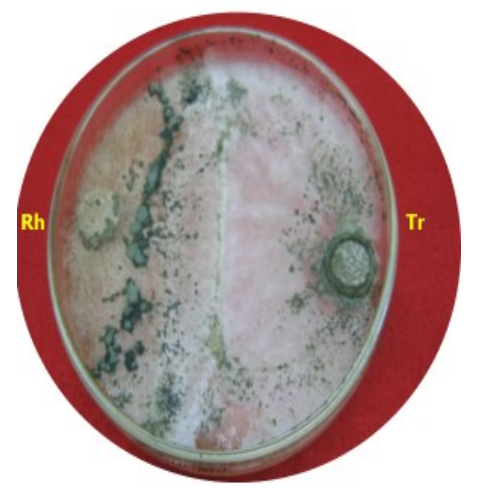

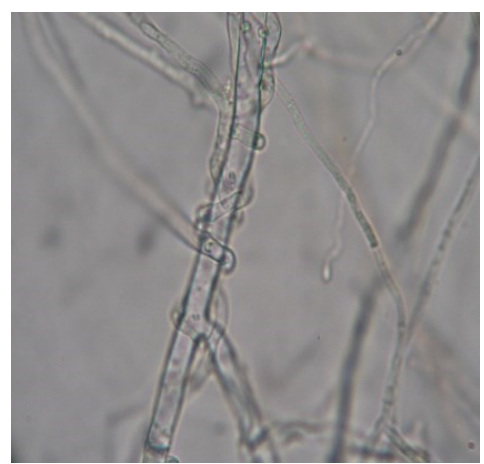

Al observar la zona de confrontación de Trichoderma con R.solani, se evidenció invasión del micelio de Rhizoctonia por Trichoderma (Fig. 2, izquierda). Cuando se observó al microscopio muestras procedentes de la zona de invasión, se constató el parasitismo de Trichoderma sobre la hifa de Rhizoctonia (Fig. 2, centro), lo cual resultó en lisis del micelio de R. solani (Fig. 2 derecha).

Figura 2.

Traslape del micelio de Trichoderma harzianum T327 sobre el micelio de Rhizoctonia solani (izquierda), hifa de R. solani parasitada por T327 (centro) y fragmentación total de micelio de R. solani debido al parasitismo de T327 (derecha).

\section{DISCUSIÓN}

Los aislamientos de Trichoderma evaluados favorecieron la germinación y emergencia de frejol Caupí alcanzando un porcentaje muy similar al obtenido en la siembra del frejol en suelo estéril que fue de $94.4 \%$. Tal hallazgo difiere con los resultados de Lucon et al. (2017), quienes refieren niveles de germinación y control de chupadera fungosa de $100 \%$ en lechuga.

La evaluación in vitro de Trichoderma sobre $R$. solani mediante el cultivo dual, permitió evidenciar el micoparastismo de los aislamientos en estudio. Al respecto, Martínez et al. (2013), en su extensa revisión sobre el género Trichoderma, refieren que el método del cultivo dual es muy utilizado para evaluar in vitro el efecto de Trichoderma, por antibiosis, la competencia por el sustrato y el micoparasitismo, aspectos en los que intervienen la velocidad Attribution-NonCommercial 4.0 International (CC BY-NC 4.0) de crecimiento de las cepas del antagonista y factores externos como tipo de sustrato, $\mathrm{pH}$ y temperatura, entre otros. Asimismo, Pavone (2012), en un estudio realizado de la acción biocontroladora de Trichoderma sp. sobre Rhizoctonia solani Khün concluye que la evaluación de las propiedades biocontroladoras de Trichoderma spp., sobre hongos fitopatógenos, a través de cultivos duales en medios sólidos, es una herramienta muy útil, pero que es recomendable complementar con ensayos de vivero y campo.

En los diferentes tratamientos de esta investigación se evidenció el efecto antagónico de Trichoderma que inhibió y mostro traslape del micelio de R. solani coincidiendo con los resultados obtenidos por Osorio et al. (2016), en su investigación sobre la actividad antagónica in vitro de Trichoderma sp. sobre tres cepas de $R$. solani (cepas J53; CH42 y HSO56), el aislamiento T18 de T. asperellum presentó el máximo efecto antagónico y creció mostrando traslape del crecimiento micelial de las tres cepas de R. 
ISSNe: 2617-9156

solani. Sin embargo, sustentan que en los ensayos realizados se observó que no todas las cepas evaluadas de Trichoderma spp. tienen la misma capacidad de inhibir el crecimiento micelial o colonizar el micelio de $R$. solani. es decir, la capacidad antagónica de Trichoderma spp. es variable, debido a que cada especie puede tener diferente mecanismo de acción; además, de la capacidad de esporulación y crecimiento.

En las observaciones al microscopio compuesto de las zonas de traslape del micelio de los hongos en estudio se constató la invasión y lisis del micelio de $R$. solani. $\mathrm{Al}$ respecto, Sánchez-García et al., (2017), sostienen que el antagonismo del género Trichoderma está dado principalmente por el parasitismo y describen que las siete cepas de $T$. harzianum y $T$. asperellum identificadas morfológicamente y molecularmente mostraron niveles diferentes de micoparasitismo en confrontaciones con los patógenos de raíz de frijol como Fusarium sp. y $R$. solani. Las hifas de $T$. asperellum (Tri-3) penetraron, formando haustorios sobre hifas de R. solani; T. harzianum (Tri-4) formó haustorios, penetró y creció como parásito dentro de hifas de $R$. solani 10 a 12 días después del inicio de la confrontación. Similar resultado fue reportado por Chávez (2006) y Hernández et al. (2011) sobre el micelio de Rhizoctonia, Sclerotinia, Fusarium y Macrophomina. López Mondéjar (2011) y González (2002), lograron también observar lisis del micelio de Phytophthora capsici al confrontar, in vitro, a este fitopatógeno con Trichoderma spp.

\section{CONCLUSIONES}

Todos los aislamientos de Trichoderma evaluados favorecieron la germinación y emergencia de frejol Caupi; asimismo, produjeron invasión y lisis del micelio de Rhizoctonia solani.

Los porcentajes más bajos de la incidencia de chupadera fungosa (3.29 y $5.25 \%)$ se obtuvieron con Trichoderma harzianum (T327) y Trichoderma asperellum (T17), constituyéndose así en una alternativa biológica promisoria, para el control de esta enfermedad.

\section{REFERENCIAS}

[1] Arcos, J., \& Zúñiga, D. (2015). Efecto de rizobacterias en el control de Rhizoctonia solani en el cultivo de papa. Ecología Aplicada, 14 (2), 95-101. Disponible en: http://www.scielo.org.pe/scielo.php?script=sci arttext \&pid $=$ S1726-22162015000200002\&lng=es\&tlng=es.

[2] Barahona, A. C. (2012). Control biológico de Rhizoctonia solani Kühn en papa (Solanum tuberosum) mediante diferentes concentraciones y formulados de una cepa de Bacillus subtilis Cohn Tesis de Pregrado. Universidad Austral, Chile. Disponible en: http://cybertesis.uach.cl/tesis/uach/2012/fab224c/doc/f ab224c.pdf

[3] Bell, D.K., Wells. H.D., \& Markham, C. R. (1982). In vitro antagonism of Trichoderma species against six fungal plant pathogens. Phytopathology, 72 (4), 379382. DOI: 10.1094/Phyto-72-379

[4] Capouz, E. R. (2009). Identificación de microorganismos antagonistas de fitopatógenos de suelo y su efecto in vitro e invernadero en especies hortícolas Tesis de Pregrado. Universidad de Guayaquil, Ecuador. Disponible en: https://www.bibliotecasdelecuador.com/Record/oai:loc alhost:28000-964

[5] Chávez, G. P. (2006). Producción de Trichoderma sp. y evaluación de su efecto en cultivo de crisantemo (Dendranthema grandiflora) Tesis de pregrado. Universidad Javeriana, Colombia. Disponible en: https://repository.javeriana.edu.co/handle/10554/8312

[6] Esparza, L. (2009). Efectividad in vitro de cepas nativas de Trichoderma spp. en aislados de Phytophthora parasítica D. obtenidos de plantas de Jamaica Tesis de Maestría. Colegio de postgraduados Montecillo, México. Disponible en: https:/www.repositorionacionalcti.mx/recurso/oai:colp osdigital.colpos.mx:10521/1357

[7] Esquivel, Z. J. (2012). Control biológico de hongos fitopatógenos de interés económico Tesis de pregrado. Universidad Nacional de Trujillo, Perú.

[8] Gonza Carnero. K., López, M. E., Zavaleta, S. C., De la Cruz, C. J. \& Mendoza, M. W. (2013). Efecto biofungicida de Trichoderma harzianum y de extractos de Eucalyptus globulus, Rosmarinus officinalis y Ricinus communis sobre Rhizoctonia solani. Rebiolest, 1(1),43-48. Disponible en: https://www.semanticscholar.org/paper/EfectobiofungicidadeTrichodermaharzianumyeyCarneroMedi na/dc50c60c8847645fbdd6b9566306cd8a684732cf

[9] González, J. E. (2002). Control biológico de Fusarium oxysporum f. sp. asparagi Schlecht: mediante bacterias antagonistas, Trichoderma spp. y humus de lombriz Tesis de maestría Universidad Nacional Agraria La Molina, Perú.

[10] Guédez, C., Castillo, C., Cañizales, L \& Olivar, R. (2009). Control biológico: una herramienta para el desarrollo sustentable y sostenible. Academia, 7(13), 50-61. Disponible en: https://www.researchgate.net/publication/236852483 Control_Biologico_Una_herramienta_para_el_desarrol lo_sustentable_y_sostenible

[11] Hernández, J.L., Sánchez, M.I., García, G.G., Pérez, N.M., González, J.M., \& Quiroz, J.D.C. (2011). Caracterización molecular y agronómica de aislados de Trichoderma spp nativos del noreste de México. Revista Colombiana de Biotecnología, 12(2), 176-185. Disponible en: 
ISSNe: 2617-9156

https://revistas.unal.edu.co/index.php/biotecnologia/art icle/view/2800

[12] Hoyos-Carvajal, L., Duque, G. y Orduz, S. (2008). Antagonismo in vitro de Trichoderma spp. sobre aislamientos de Sclerotinia spp. y Rhizoctonia spp. Revista Colombiana de Ciencias Hortícolas, 2(1) ,7686. DOI: https://doi.org/10.17584/rcch.2008v2i1.1175

[13] Infante, D., González, N., \& Reyes, Y. (2010). Mecanismos de acción de Trichoderma frente a hongos fitopatógenos. Revista de Protección Vegetal, 24(1),34$38 . \quad$ Disponible http://scielo.sld.cu/pdf/rpv/v24n1/rpv02109.pdf

[14] Infante, D., Martínez, B., Pereira, B., Reyes, Y., \& Herrera, A. (2013). Identificación molecular y evaluación patogénica de trece aislamientos de Trichoderma spp. frente a Rhizoctonia solani Kühn. Biotecnología Aplicada, 30,17-22. Disponible en: https://www.medigraphic.com/cgi$\mathrm{bin} / \mathrm{new} /$ resumen.cgi?IDARTICULO $=48178$

[15] López Mondéjar. R. (2011). Evaluación de su actividad biocontrol frente a la fusariosis vascular del melón mediante la aplicación de herramientas moleculares Tesis de Doctorado. Universidad de Alicante, España.

[16] Lucon, C.M.M., Tivelli, S. W., Yoshizumi, M. (Abril del 2017). Estimulación del crecimiento de plántulas de lechuga $\mathrm{y}$ control del damping off causado por Rhizoctonia solani con Trichoderma spp. Control Biológico. Simposio llevado a cabo en el VIII Seminario Científico Internacional del Instituto de Investigaciones de Sanidad Vegetal. La Habana, Cuba.

[17] Martínez, B., Infante, D., \& Reyes, Y. (2013). Trichoderma sp. y su función en el control de plagas en los cultivos. Revista de Protección Vegetal, 28 (1),1-11. Disponible en: http://scielo.sld.cu/scielo.php?script=sci_arttext\&pid= S1010-27522013000100001

[18] Michel, A. A. (2001). Cepas nativas de Trichoderma spp (Euascomycetes: Hypocreales), su antibiosis y micoparasitismo sobre Fusarium subglutinans y Fusarium oxysporum (Hyphomycetes: Hyphales) Tesis de Doctorado. Universidad de Colima, México. Disponible

en: https://sistemas.ucol.mx/tesis_posgrado/resumen 1297 . htm

[19] Osorio, E., Hernández, F.D., Rodríguez, R., Varela, S.E., Estrada, B., \& López, J. A. (2016). Actividad antagónica de Trichoderma spp. sobre Rhizoctonia solani in vitro. Investigación y Ciencia, 24 (67), 5-11. Disponible https://www.redalyc.org/pdf/674/67446178001.pdf
[20] Pavone, D.F. (2012). Biocontrol de Rhizoctonia solani Khün por Trichoderma spp. Tesis de Doctorado. Universidad Central de Venezuela, Caracas.

[21] Reyes, Y., Infante, D., \& Martínez, B. (2016). Eficacia de Trichoderma asperellum Samuels, Lieckfeldt \& Nirenberg para el control de Rhizoctonia solani Kühn en condiciones de campo. Revista de Protección Vegetal, 31(2), 107-113. Disponible en http://scielo.sld.cu/scielo.php?script=sci_arttext\&pid= S1010-27522016000200005

[22] Rubio, R. G., Baltodano, S. F., Abanto, C. L., Wilson, K. J. \& Muñoz, R. M. (2008). Resistencia in vitro de Rhizoctonia solani y Fusarium oxysporum a los fungicidas Benzomil 500, Rhizolex-T y Homai-WP. Revista Biológica de la Universidad de Trujillo, Perú. 28 (2), 34-46. Disponible en: https://es.scribd.com/document/63156308/Resist-enCIA-in-Vitro-de-Rhizoctonia-Solani-y-FusariumOxysporum-a-Los-Fungi-Cid-As-Benzomil-500Rhizolex-T-y-Homai-WP

[23] Sánchez-García, B.M., Espinosa-Huerta, E., VillordoPineda, E., Rodríguez-Guerra, R. \& Mora-Avilés, M. A. (2017). Identificación molecular y evaluación antagónica in vitro de cepas nativas de Trichoderma spp. sobre hongos fitopatógenos de raíz en frijol (Phaseolus vulgaris L.) cv. montcalm. Agrociencia, 51(1), 63-79. Disponible en: http://www.scielo.org.mx/scielo.php?script=sci_arttext \&pid $=\mathrm{S} 1405-31952017000100063 \& \operatorname{lng}=\mathrm{es} \& \mathrm{nrm}=$ iso

[24] Tovar, C. J. (2008). Evaluación de la capacidad antagónica "in vivo" de aislamientos de Trichoderma spp. frente al hongo fitopatógeno Rhizoctonia solani Tesis de Pregrado. Pontificia Universidad Javeriana, Colombia. Disponible en: https://repository.javeriana.edu.co/handle/10554/8951 